\title{
Admissibility region for rarefaction shock waves in dense gases
}

\author{
CALIN ZAMFIRESCU ${ }^{1}$, ALBERTO GUARDONE \\ AND PIERO COLONNA ${ }^{1}$ \\ ${ }^{1}$ Process and Energy Department, Delft University of Technology, Leghwaterstraat 44, \\ Delft, $2628 \mathrm{CA}$, The Netherlands \\ ${ }^{2}$ Dipartimento di Ingegneria Aerospaziale, Politecnico di Milano, Via La Masa 34, \\ Milano, 20154, Italy
}

(Received 19 April 2007 and in revised form 5 December 2007)

In the vapour phase and close to the liquid--vapour saturation curve, fluids made of complex molecules are expected to exhibit a thermodynamic region in which the fundamental derivative of gasdynamic $\Gamma$ is negative. In this region, non-classical gasdynamic phenomena such as rarefaction shock waves are physically admissible, namely they obey the second law of thermodynamics and fulfil the speed-orienting condition for mechanical stability. Previous studies have demonstrated that the thermodynamic states for which rarefaction shock waves are admissible are however not limited to the $\Gamma<0$ region. In this paper, the conditions for admissibility of rarefaction shocks are investigated. This results in the definition of a new thermodynamic region - the rarefaction shocks region - which embeds the $\Gamma<0$ region. The rarefaction shocks region is bounded by the saturation curve and by the locus of the states connecting double-sonic rarefaction shocks, i.e. shock waves in which both the pre-shock and post-shock states are sonic. Only one double-sonic shock is shown to be admissible along a given isentrope, therefore the double-sonic states can be connected by a single curve in the volume-pressure plane. This curve is named the double sonic locus. The influence of molecular complexity on the shape and size of the rarefaction shocks region is also illustrated by using the van der Waals model; these results are confirmed by very accurate multi-parameter thermodynamic models applied to siloxane fluids and are therefore of practical importance in experiments aimed at proving the existence of rarefaction shock waves in the single-phase vapour region as well as in future industrial applications operating in the non-classical regime.

\section{Introduction}

The classical theory of gasdynamic discontinuities, see e.g. Hayes (1960), moves from the conservation laws in the shock reference frame to derive the well-known RankineHugoniot equation which relates the pressure $P$ and the specific volume $v$ past the shock wave for given values of $P$ and $v$ ahead of it. The observation - valid for the case of a constant-specific-heat (polytropic) ideal gas - that the curvature of the RankineHugoniot locus is always concave up, together with the condition that the specific entropy $s$ must increase across the shock, leads to the conclusion that physically admissible shocks are of the compression type only, whereas rarefaction shock waves are not admissible. This results from noting that, for a polytropic ideal gas, isentropes are always concave-up in the $(v, P)$-plane and so are the Rankine-Hugoniot 


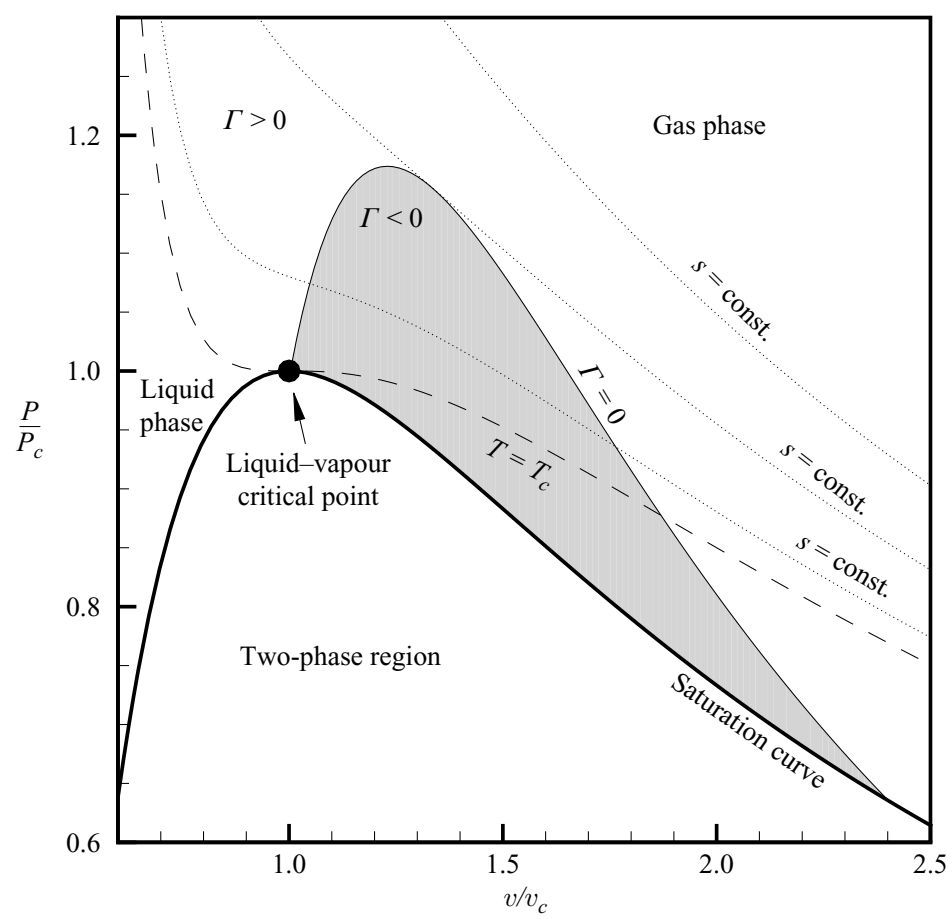

FIGURE 1. Liquid-vapour saturation curve and $\Gamma<0$ region (shaded region) for a BZT fluid in the volume-pressure plane computed from the polytropic van der Waals model with $R / c_{v}=0.001$. Selected isentropes and the critical isotherm $T=T_{\mathrm{c}}$ are also indicated. Note that the isentropes are concave-down in the $\Gamma<0$ region.

curves, see Godlewski \& Raviart (1994). The above conclusions also remain valid in most gasdynamic applications of practical interest, including shock waves occurring in chemically reacting flows or detonation waves.

However, the upward concavity of the isentropes is not the consequence of any thermodynamic constraint and modern thermodynamic models of fluids characterized by high molecular complexity - named Bethe-Zel'dovich-Thompson (BZT) fluids predict isentropes with downward curvature in a limited region in the vapour phase and close to the liquid-vapour saturation curve, see figure 1. A non-dimensional measure of the curvature of the isentropes has been introduced by Thompson (1971) and it is usually referred to as the fundamental derivative of gasdynamics $\Gamma$, defined as

$$
\Gamma \equiv \frac{v^{3}}{2 c^{2}}\left(\frac{\partial^{2} P}{\partial v^{2}}\right)_{s}
$$

where $c^{2}, c^{2}=-v^{2}(\partial P / \partial v)_{s}$, is the speed of sound and where, according to standard thermodynamic nomenclature, the subscript $s$ indicates that the pressure derivative is computed at constant entropy. Flows evolving in the $\Gamma<0$ region may significantly differ from their polytropic ideal-gas counterparts. For example, a rarefaction shock wave (RSW) characterized by two state-points with $\Gamma<0$ is physically admissible, since the curvature of the isentrope and hence of the Rankine-Hugoniot curve between the two states is reversed (negative) with respect to the ideal gas case, as pointed out by Bethe (1942), Zel'dovich (1946) and other authors. Landau \& Lifshitz (1959) proved the following relation between the entropy difference $\Delta s$ and the specific 
volume difference $\Delta v$ across a shock wave

$$
\Delta s=-\frac{\Gamma_{\mathrm{A}}}{6} \frac{c_{\mathrm{A}}^{2}}{T_{\mathrm{A}}}\left(\frac{\Delta v}{v_{\mathrm{A}}}\right)^{3}+O\left([\Delta v]^{4}\right),
$$

where $T$ is the temperature and where the subscript A indicates the pre-shock state. The above relation has been derived by Taylor expansion centred on $\mathrm{A}$ and it is only applicable to weak shock waves. The weak shock theory therefore identifies compressive $(\Delta v<0)$ shocks as physically admissible shocks $(\Delta s>0)$ if $\Gamma>0$, whereas $\operatorname{RSW}(\Delta v<0)$ are possible only if $\Gamma<0$, see also Thompson (1971). Mixed waves, such as for example a mixed rarefaction shock-fan combination, can occur if the $\Gamma=0$ boundary is crossed during flow evolution, a likely situation due to the limited extent of the $\Gamma<0$ region. Rarefaction shocks and mixed waves are usually referred to as non-classical waves, to distinguish them from the (classical) compression shocks and isentropic rarefaction waves.

The importance of the sign of $\Gamma$ led researchers to identify the $\Gamma<0$ region as the so-called non-classical gasdynamic region, with the understanding that all flows evolving within the $\Gamma$-negative thermodynamic region have opposite features with respect to their classical counterparts. This definition is somewhat misleading, in that, as pointed out by Fergason et al. (2001), an admissible RSW may also occur between two states characterized by $\Gamma>0$, provided that the corresponding RankineHugoniot curve crosses the $\Gamma=0$ boundary. A similar result has been illustrated by Thompson \& Lambrakis (1973) under the assumption of isentropic shocks of finite strength. The existence of such waves, which cannot be directly explained by resorting to the weak shock theory of gasdynamics, points out the limitations of the current definition of the non-classical region.

In this work, the admissibility conditions for the formation of an RSW are studied in order to identify the thermodynamic region in which RSWs can occur. A new rarefaction shocks region (RSR) is therefore introduced. This is bounded by the liquid-vapour saturation curve and a new thermodynamic curve named the double sonic locus (DSL), connecting state points from which a double sonic shock - a shock wave with both pre-shock and post-shock sonic state, see e.g. Thompson \& Lambrakis (1973) and Cramer \& Sen (1987) - can originate. The shape and extent of the RSR is studied by means of the simple polytropic van der Waals model for fluids of increasing molecular complexity. These qualitative results are confirmed by the more accurate multi-parameter Span-Wagner thermodynamic models applied to siloxane fluids.

The identification of the shape of the RSR is of theoretical as well as practical interest, because it can be directly used to determine the most suitable operating conditions for industrial applications taking advantage of the peculiarities of BZT fluids. Moreover, owing to the relatively small extent of the non-classical region, with pressure and temperature spanning only few bars and degrees Kelvin, respectively, see Guardone \& Argrow (2005), the correct identification of the boundaries of this region is of paramount importance prior to any experimental attempt to demonstrate the existence of non-classical phenomena in the vapour phase. No generally accepted experimental evidence of such phenomena is currently available. The results of this work have been used in the design of a dense gas Ludwieg tube for the generation of rarefaction shock waves in siloxanes, see Zamfirescu, Guardone \& Colonna $(2006 a, b)$.

This paper is structured as follows. In $\S 2$, the Rankine-Hugoniot jump conditions relating the flow states across a discontinuity are briefly recalled and the admissibility condition for a shock wave is discussed. Different scenarios for the formation of an 
RSW are illustrated. In $\S 3$, the definition of the RSR is given and the influence of molecular complexity on the shape and extent of the RSR is investigated using the simple van der Waals thermodynamic model. These results are confirmed by more accurate multi-parameter thermodynamic models for siloxanes. Section 4 outlines the concluding remarks.

\section{Rarefaction shock waves in dense gases}

This section briefly addresses the flow conditions resulting in the formation of a nonclassical rarefaction shock wave, in terms of both the integral relations governing the flow states across the discontinuity and the admissibility conditions. Shock adiabats of different shape are discussed to identify the thermodynamic region in which rarefaction shock waves are possible, namely the RSR, and the definition of the double sonic locus (DSL) is given.

\subsection{Jump relations and the admissibility condition}

Rarefaction and compression shock waves are discontinuous solutions to the Euler equation of gasdynamics and these waves are therefore required to satisfy the wellknown Rankine-Hugoniot jump relations linking the two states A and B separated by the shock. In the following $\mathrm{A}$ indicates the pre-shock state, while $\mathrm{B}$ indicates the candidate post-shock state. The Rankine-Hugoniot relations are obtained by writing the integral form of the governing equations in the shock-reference frame, a reference frame moving with the velocity of the discontinuity, see e.g. Hayes (1960). These are

$$
\left.\begin{array}{rl}
u_{\mathrm{A}} / v_{\mathrm{A}} & =u_{\mathrm{B}} / v_{\mathrm{B}}, \\
P_{\mathrm{A}}+u_{\mathrm{A}}^{2} / v_{\mathrm{A}} & =P_{\mathrm{B}}+u_{\mathrm{B}}^{2} / v_{\mathrm{B}}, \\
h\left(P_{\mathrm{A}}, v_{\mathrm{A}}\right)+\frac{1}{2} u_{\mathrm{A}}^{2} & =h\left(P_{\mathrm{B}}, v_{\mathrm{B}}\right)+\frac{1}{2} u_{\mathrm{B}}^{2},
\end{array}\right\}
$$

where the enthalpy $h$ is computed from the pressure and the specific volume via the thermodynamic equation of state as $h=h(P, v)$. The system of equations (2.1) allows one to compute the post-shock state B for a given pre-shock condition A. The above relations include also contact surfaces, i.e. particular discontinuities in which $P_{\mathrm{A}}=P_{\mathrm{B}}$ and $u_{\mathrm{A}}=u_{\mathrm{B}}$. As it is known, the Rankine-Hugoniot system (2.1) can be rewritten as a scalar nonlinear equation for the post-shock specific volume $v_{\mathrm{B}}$, for given values of the pre-shock state variables $v_{\mathrm{A}}, P_{\mathrm{A}}$ and $u_{\mathrm{A}}$, as follows. For a generic post-shock state $v=v_{\mathrm{B}}$ and $P=P_{\mathrm{B}}$, from (2.1) one has

$$
\mathscr{H}\left(P, v ; P_{\mathrm{A}}, v_{\mathrm{A}}\right)=h(P, v)-h\left(P_{\mathrm{A}}, v_{\mathrm{A}}\right)-\frac{1}{2}\left(P-P_{\mathrm{A}}\right)\left(v_{\mathrm{A}}+v\right)=0 .
$$

The equation $\mathscr{H}\left(P, v ; P_{\mathrm{A}}, v_{\mathrm{A}}\right)=0$ provides an implicit definition of the RankineHugoniot curve or shock adiabat centred on A, i.e.

$$
P(v)=P^{\mathrm{H}}\left(v ; P_{\mathrm{A}}, v_{\mathrm{A}}\right),
$$

which gives the post-shock value of the pressure as a function of the post-shock specific volume for a given pre-shock thermodynamic state A. Note that the velocity $u$ does not appear in the expression for $P^{\mathrm{H}}$, which is a purely thermodynamic relation. By combining the conservation of mass and momentum in system (2.1), the Rayleigh line is obtained as

$$
P(v)=P^{\mathrm{R}}\left(v ; P_{\mathrm{A}}, v_{\mathrm{A}}, u_{\mathrm{A}}\right)=P_{\mathrm{A}}+\left(1-\frac{v}{v_{\mathrm{A}}}\right) \frac{u_{\mathrm{A}}^{2}}{v_{\mathrm{A}}} .
$$




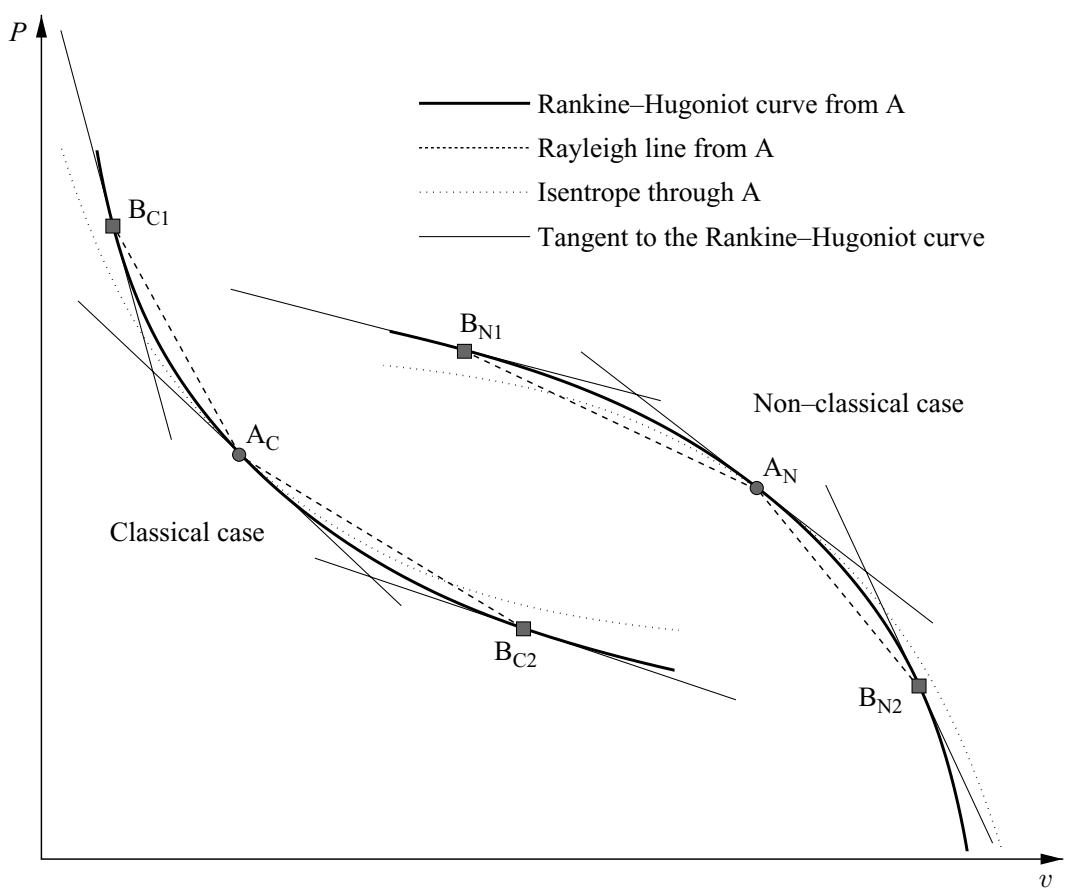

FiguRE 2. Geometrical interpretation of the admissibility condition (2.6). Points $A_{C}$ and $A_{N}$ are the pre-shock states for the classical and non-classical case; possible post-shock states are indicated with the letter $\mathrm{B}$.

The downstream specific volume $v_{\mathrm{B}}$ is finally found by solving for $v_{\mathrm{B}}$ the equation

$$
P^{\mathrm{H}}\left(v_{\mathrm{B}} ; P_{\mathrm{A}}, v_{\mathrm{A}}\right)=P^{\mathrm{R}}\left(v_{\mathrm{B}} ; P_{\mathrm{A}}, v_{\mathrm{A}}, u_{\mathrm{A}}\right),
$$

which in general admits multiple solutions.

The unique admissible downstream state among the solutions of (2.5) is selected by imposing the entropy condition $\Delta s>0$, see Smoller (1983) and Oleinik (1959), which, together with the shock stability or speed orienting condition $M_{\mathrm{A}} \geqslant 1 \geqslant M_{\mathrm{B}}$, with $M=|u| / c$ the Mach number, see Lax (1957), gives

$$
\left.\frac{\mathrm{d} P^{\mathrm{H}}}{\mathrm{d} v}\right|_{\mathrm{A}} \geqslant\left.\left.\frac{\mathrm{d} P^{\mathrm{R}}}{\mathrm{d} v}\right|_{\mathrm{A}} \equiv \frac{P_{\mathrm{A}}-P_{\mathrm{B}}}{v_{\mathrm{A}}-v_{\mathrm{B}}} \equiv \frac{\mathrm{d} P^{\mathrm{R}}}{\mathrm{d} v}\right|_{\mathrm{B}} \geqslant\left.\frac{\mathrm{d} P^{\mathrm{H}}}{\mathrm{d} v}\right|_{\mathrm{B}},
$$

where equalities hold for sonic pre- or post-shock states, see Kluwick (2001). The above expression allows a geometrical interpretation as explained in the following.

With reference to figure 2 , the classical gasdynamic case is analysed first. Consider a state point $\mathrm{A}_{\mathrm{C}}$ in the $(v, P)$-plane. The isentrope through $\mathrm{A}_{\mathrm{C}}$ is concave up since $\Gamma>0$ everywhere. Correspondingly, the Rankine-Hugoniot curve from point $\mathrm{A}_{\mathrm{C}}$ is concave up, since it shares a second-order contact point with the isentrope in $\mathrm{A}_{\mathrm{C}}$, as shown in the following. Differentiating equation (2.2), $\mathrm{d} \mathscr{H}=0$, and substituting in the expression obtained the definition of the enthalpy $h(P, v)=e(P, v)+P v$, where $e$ is the internal specific energy per unit mass, and the thermodynamic identity $T \mathrm{~d} s=\mathrm{d} e+P \mathrm{~d} v$, yields

$$
2 T \mathrm{~d} s=\left(P-P_{\mathrm{A}}\right) \mathrm{d} v-\left(v-v_{\mathrm{A}}\right) \mathrm{d} P,
$$


which gives $\mathrm{d} s \equiv 0$ at $\mathrm{A}$. The Rankine-Hugoniot curve is therefore tangent in A to the isentrope through A. Differentiating again, one obtains

$$
2 T \mathrm{~d}^{2} s+2(\mathrm{~d} T) \mathrm{d} s=\left(P-P_{\mathrm{A}}\right) \mathrm{d}^{2} v-\left(v-v_{\mathrm{A}}\right) \mathrm{d}^{2} P,
$$

which gives $\mathrm{d}^{2} s \equiv 0$ at $\mathrm{A}$, that is, the Rankine-Hugoniot curve and the isentrope through A are osculatory at A, see Godlewski \& Raviart (1994).

Two possible post-shock states $\mathrm{B}_{\mathrm{C} 1}$ and $\mathrm{B}_{\mathrm{C} 2}$ are now considered. Point $\mathrm{B}_{\mathrm{C} 1}$, which corresponds to a compression shock, is admissible since it satisfies the admissibility criterion (2.6). The slope $\mathrm{d} P^{\mathrm{H}} / \mathrm{d} v$ of the Rankine-Hugoniot curve at point $\mathrm{A}_{\mathrm{C}}$ is larger than the slope of the Rayleigh line $\mathrm{A}_{\mathrm{C}}-\mathrm{B}_{\mathrm{C} 1}$, which in turn is greater than the slope $\mathrm{d} P \mathrm{H} / \mathrm{d} v$ in $\mathrm{B}_{\mathrm{C} 1}$, as prescribed by condition (2.6). Conversely, the rarefaction shock $\mathrm{A}_{\mathrm{C}}-\mathrm{B}_{\mathrm{C} 2}$ is not admissible. The reversed situation occurs in the non-classical case, where both the isentropes and the Rankine-Hugoniot curve are concave-down and therefore admissible shock waves are solely of the rarefaction type.

Criterion (2.6) provides a useful tool to identify the admissible shocks along a given shock adiabat, by simple comparison of the slope of the adiabat at the preand post-shock states with the slope of the segment connecting the pre-shock state. Criterion (2.6) is used in the next section to determine the admissibility conditions for a non-classical RSW in the various possible situations occurring in the compressible flow of a BZT fluid.

\subsection{Rarefaction shock waves}

It is remarkable that the Rankine-Hugoniot curve (2.3) identifies a two-parameter family of thermodynamic curves, differently from more common thermodynamic loci such as, for example, isotherms or isentropes, which are defined by one single parameter. Each shock adiabat is defined by specifying the pre-shock values of both the pressure and the specific volume independently - or of any two other independent thermodynamic variables identifying the pre-shock thermodynamic state of the fluid.

Inspection of figure 1 reveals that for entropies larger than the special value $s_{\tau}$, for which the isentrope is tangent to the $\Gamma=0$ line, the isentropes and hence the Rankine-Hugoniot curves are concave-up everywhere, and the classical situation is recovered. Isentropes with $s<s_{\tau}$ cross the $\Gamma=0$ line twice and therefore a portion of these isentropic curves exhibits a downward curvature. Isentropes intersecting the vapour-liquid equilibrium (VLE) line are not considered since this study is limited to the treatment of single-phase flows. Single-phase isentropes with $s<s_{\tau}$ display one and the same qualitative shape and the study of the Rankine-Hugoniot curve can be accomplished by considering initial states located along a representative isentrope crossing the $\Gamma=0$ line.

With reference to figure 3 , four different state points with increasing specific volumes, namely points $\mathrm{A}_{1}, \mathrm{~A}_{2}, \mathrm{~A}_{3}$ and $\mathrm{A}_{4}$, are considered in order to identify the different scenarios for the occurrence of an RSW. All these points are located along the isentrope $s=s_{\mathrm{A}}<s_{\tau}$. Point $\mathrm{A}_{4}$ is characterized by $\Gamma<0$, whereas $\Gamma$ is positive for all remaining points. Each state point is taken as the pre-shock state to construct the corresponding Rankine-Hugoniot curves in figures 4-7, where candidate post-shock points $\mathrm{B}$ are also indicated. Note that the curves in figures 4-7 are only qualitative, used here only for explanation purposes. Points $A_{1}, A_{2}, A_{3}$ and $A_{4}$ have been chosen in order to investigate all the possible conditions for the formation of an RSW. It is anticipated here, to the understanding of the following treatment, that from point $\mathrm{A}_{1}$ no RSW is admissible, that only a double sonic RSW can originate from $\mathrm{A}_{2}$ and that 


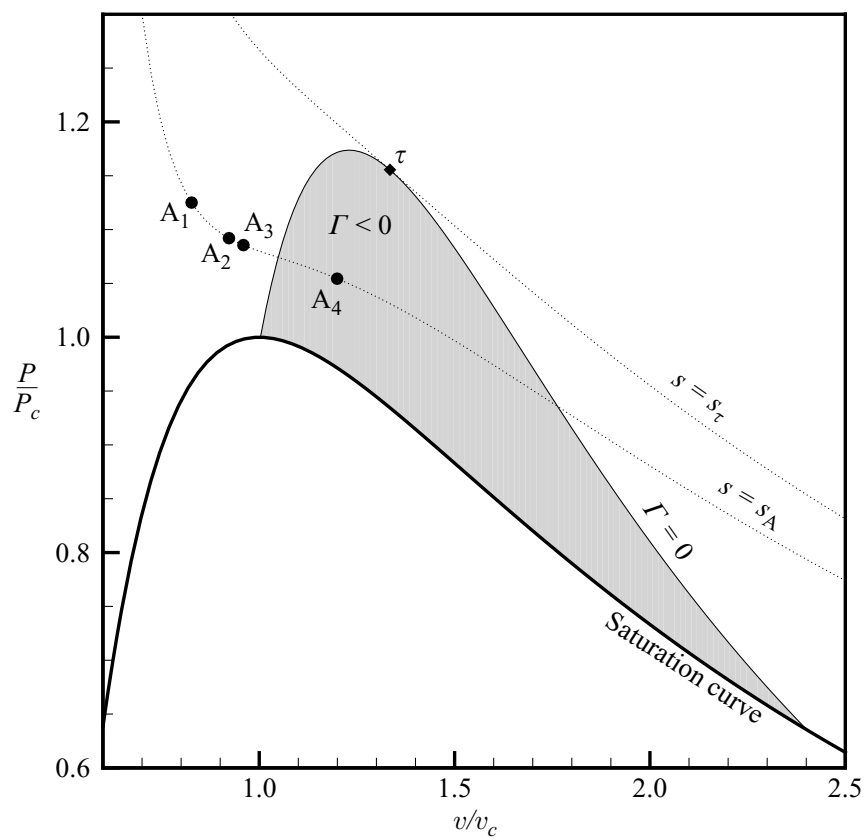

FiguRE 3. Selected pre-shock state points along a representative isentrope $s=s_{\mathrm{A}}<s_{\tau}$. The corresponding Rankine-Hugoniot curves are drawn in figures 4-7.

a pre- and post-shock sonic RSW as well as non-sonic RSW can originate from point $\mathrm{A}_{3}$. Finally, from point $\mathrm{A}_{4}$, only post-shock sonic and non-sonic RSW can arise.

State $A_{1}$ in figure 4 is considered first. Since $\Gamma>0$ at $A_{1}$, both the isentrope through $A_{1}$ and the Rankine-Hugoniot curve centred at $A_{1}$ are concave-up at $A_{1}$, see $\S 2.1$. Figure 4 qualitatively shows both the Rankine-Hugoniot curve and the isentrope through $A_{1}$. Post-shock state $B_{11}$ fulfils the entropy condition (2.6) and therefore the classical compression shock $A_{1}-B_{11}$ is admissible. The above does not hold for the remaining candidate post-shock states $\mathrm{B}_{12}, \mathrm{~B}_{13}, \mathrm{~B}_{14}$ and $\mathrm{B}_{15}$, which would lead to an $\mathrm{RSW}$, as can be verified from figure 4. For these reasons, any shock originating from state $\mathrm{A}_{1}$ can be of the compressive type only.

Point $\mathrm{A}_{2}$ in figure 5 is now considered. As in the previous case, post-shock state $\mathrm{B}_{21}$ is associated with an admissible compressible shock and all states along the Rankine-Hugoniot through $\mathrm{A}_{2}$ and characterized by $v_{\mathrm{B}}<v_{\mathrm{A}_{2}}$ can be connected to $\mathrm{A}_{2}$ by a compression shock wave. On the other hand, RSW connecting states $\mathrm{B}_{22}$ and $\mathrm{B}_{23}$ do not fulfil condition (2.6). The only admissible RSW is $\mathrm{A}_{2}-\mathrm{B}_{24}$, which is a special one: at both $\mathrm{A}_{2}$ and $\mathrm{B}_{24}$, the Rayleigh line is tangent to the Rankine-Hugoniot curve, namely,

$$
\left.\frac{\mathrm{d} P_{\mathrm{H}}}{\mathrm{d} v}\right|_{\mathrm{A}}=\frac{P_{\mathrm{A}}-P_{\mathrm{B}}}{v_{\mathrm{A}}-v_{\mathrm{B}}}=\left.\frac{\mathrm{d} P_{\mathrm{H}}}{\mathrm{d} v}\right|_{\mathrm{B}},
$$

or $M_{\mathrm{A}}=1=M_{\mathrm{B}}$, that is, both the pre- and the post-shock states are sonic. This special shock wave is termed the double sonic shock and has been studied by Cramer \& Sen (1987) for a polytropic van der Waals gas. All post-shock states to the right of point $\mathrm{B}_{24}$ are not admissible. Therefore, only the double sonic RSW $\mathrm{A}_{2}-\mathrm{B}_{24}$ can be associated with point $\mathrm{A}_{2}$. 


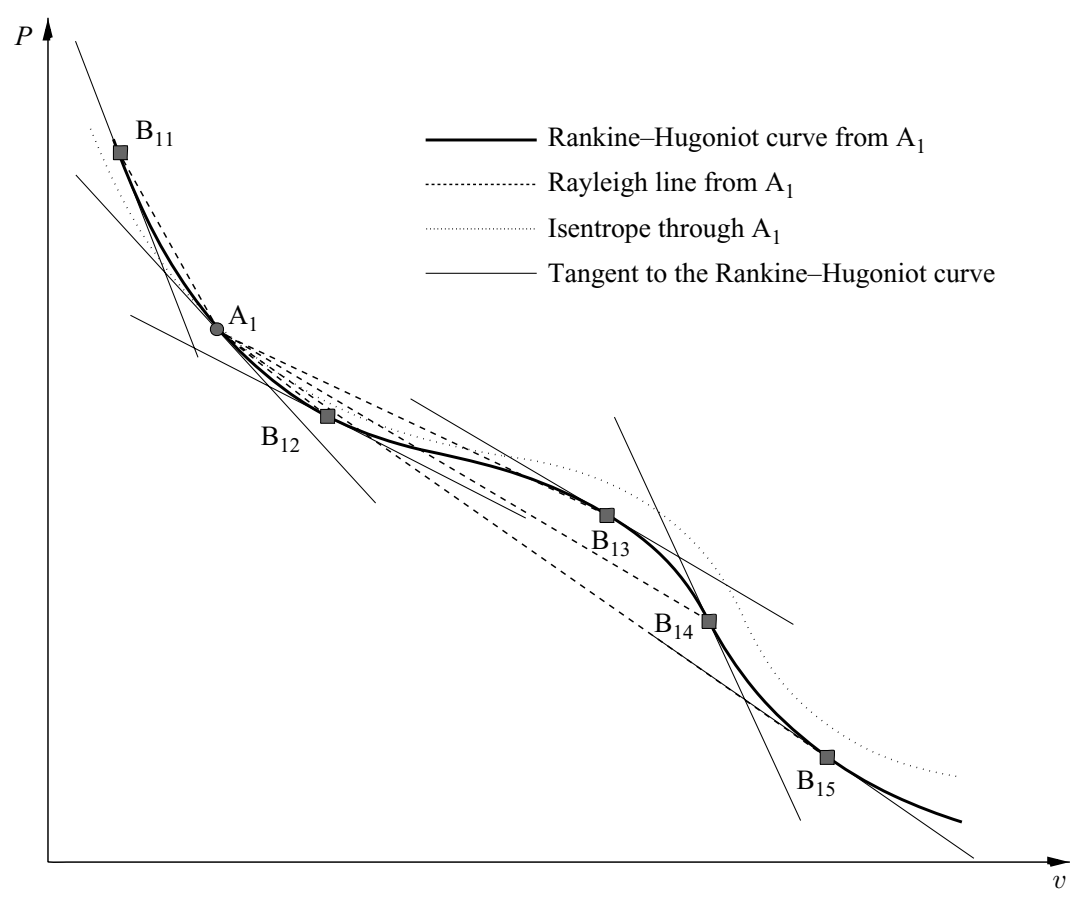

FigURE 4. Rankine-Hugoniot curve through point $A_{1}$ and candidate post-shock states $B_{11}$, $\mathrm{B}_{12}, \mathrm{~B}_{13}, \mathrm{~B}_{14}$ and $\mathrm{B}_{15}$. Only state $\mathrm{B}_{11}$ fulfils condition (2.6) and hence only a compression shock can originate from $A_{1}$.

With reference to figure 6 , admissible RSW originating from point $A_{3}$ are bounded by the two special shocks $A_{3}-B_{32}$ and $A_{3}-B_{34}$. The former is characterized by a sonic pre-shock state, since the Rayleigh line connecting points $\mathrm{A}_{3}$ and $\mathrm{B}_{32}$ is tangent to the Rankine-Hugoniot curve in $\mathrm{A}_{3}$ and hence $M_{\mathrm{A}_{3}}=1$. The latter exhibits a post-shock sonic point, with the Rayleigh line being tangent to the Rankine-Hugoniot curve in $\mathrm{B}_{34}$, where $M_{\mathrm{B}_{34}}=1$. Rarefaction shock waves connecting point $\mathrm{A}_{3}$ with a post-shock state located between points $\mathrm{A}_{3}$ and $\mathrm{B}_{32}$, as well as those having states with $v>v_{\mathrm{B}_{34}}$ are not admissible. The only admissible post-shock states allowing RSW are therefore located in between points $\mathrm{B}_{32}$ and $\mathrm{B}_{34}$ along the Rankine-Hugoniot curve, such as e.g. point $\mathrm{B}_{33}$.

Point $\mathrm{A}_{4}$ is finally considered. Since $\Gamma<0$ in $\mathrm{A}_{4}$, the curvature of the RankineHugoniot curve is negative in $\mathrm{A}_{4}$, see figure 7. All post-shock states located between points $A_{4}$ and $B_{43}$ result in admissible RSW. The limiting shock $A_{4}-B_{43}$ is sonic at $\mathrm{B}_{43}$.

No RSW can originate from fluid states featuring positive $\Gamma$ but with specific volume larger than those in the $\Gamma<0$ region. The reader is referred to Kluwick (2001) for a more detailed description, which also includes mixed waves.

\subsection{The double sonic locus (DSL)}

It can be inferred from the previous section that double sonic shocks (DSS) represent the boundary between non-classical rarefaction shocks and classical isentropic expansions. Along each isentrope there exist special state points such as $\mathrm{A}_{2}$ in figure 5 from which a double sonic RSW can originate. No other RSW is admissible from $\mathrm{A}_{2}$. 


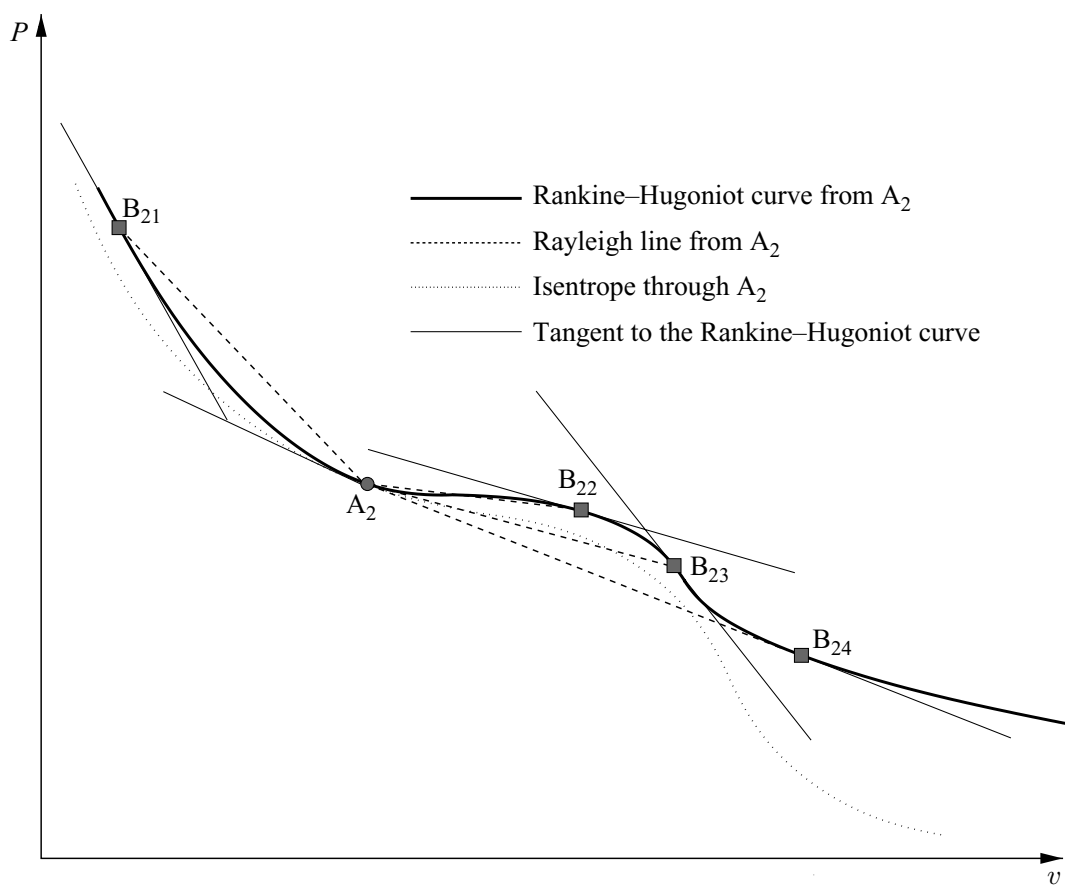

FiguRE 5. Rankine-Hugoniot curve through point $\mathrm{A}_{2}$ and candidate post-shock states $\mathrm{B}_{21}$, $\mathrm{B}_{22}, \mathrm{~B}_{23}$, and $\mathrm{B}_{24}$. States $\mathrm{B}_{21}$ and $\mathrm{B}_{24}$ fulfil condition (2.6), whereas states $\mathrm{B}_{22}$ and $\mathrm{B}_{23}$ do not correspond to a physically admissible rarefaction shock. The rarefaction shock $\mathrm{A}_{2}-\mathrm{B}_{24}$ is a double sonic shock.

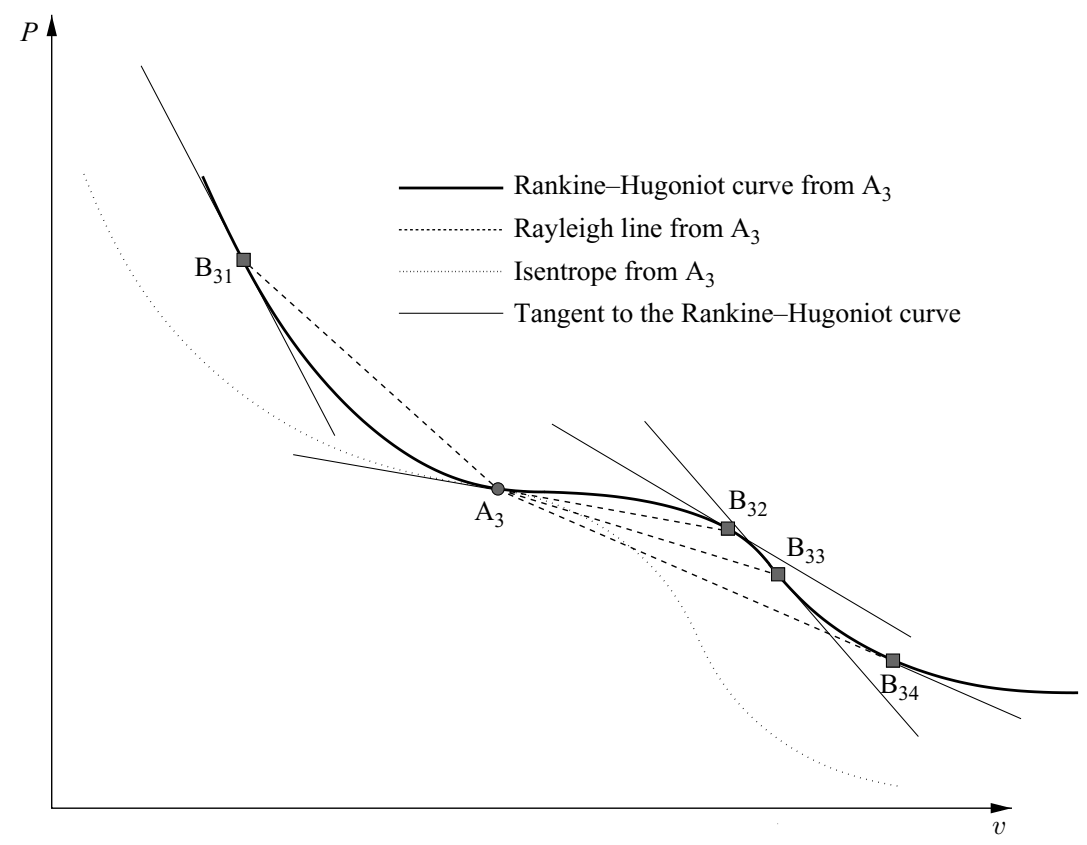

FiguRE 6. Rankine-Hugoniot curve through point $A_{3}$ and candidate post-shock states $B_{31}$, $B_{32}, B_{33}$, and $B_{34}$. The rarefaction shock $A_{3}-B_{32}$ is such that $A_{3}$ is a sonic point. The post-shock condition $\mathrm{B}_{34}$ is also sonic. 


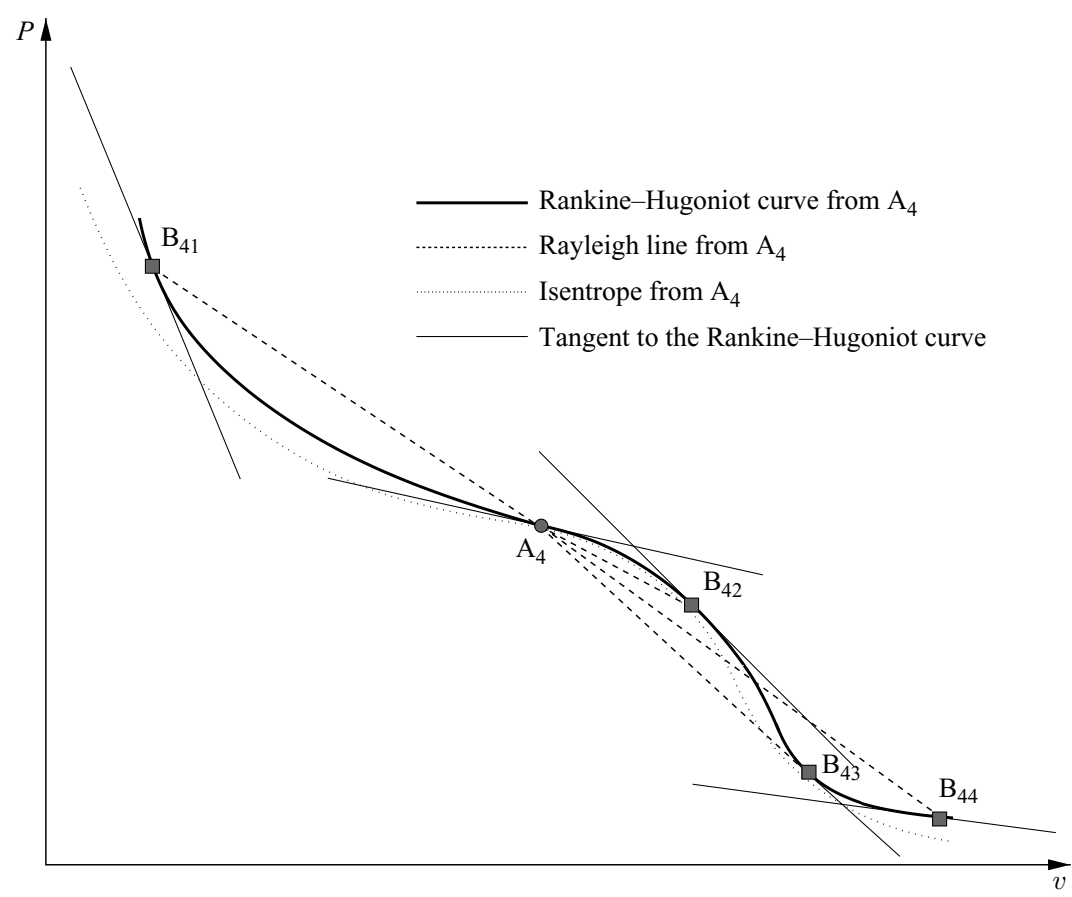

FIGURE 7. Rankine-Hugoniot curve through point $\mathrm{A}_{4}$ and candidate post-shock states $\mathrm{B}_{41}$, $B_{42}, B_{43}$, and $B_{44}$. The rarefaction shock $A_{3}-B_{43}$ is such that $B_{43}$ is sonic.

These points are labelled in the following as $A_{D}$, with $B_{D}$ being the corresponding post-shock sonic points.

Next, the uniqueness of the DSS on a given isentrope is discussed. For this purpose, figure 8 shows an expansion wave from state 1 and state 2 : the wave states are shown in the $(v, P)$ thermodynamic plane, while the wave structure is displayed in the $(x, t)$ diagram. States 1 and 2 are chosen far enough from the $\Gamma<0$ region so that the expansion wave is a mixed wave with isentropic rarefaction from state 1 to state $A_{D}$, a DSS from $A_{D}$ to $B_{D}$ and a tailing isentropic rarefaction from $B_{D}$ to point 2, see e.g. Kluwick (2001). Note that $s_{1}=s_{\mathrm{AD}_{\mathrm{D}}}$ and that $s_{2}=s_{\mathrm{BD}}$, but $s_{1} \neq s_{2}$ because of the entropy jump across the DSS. Consider a second state $A_{D}^{\prime} \neq A_{D}$ along the isentrope $s=s_{1}$, connected to the post-shock state $\mathrm{B}_{\mathrm{D}}^{\prime}$ via a DSS. Since the slope of the isentrope at $A_{D}^{\prime}$ is different from that in $A_{D}$, the slopes of the Rayleigh lines $A_{D}-B_{D}$ and $A_{D}^{\prime}-B_{D}^{\prime}$ differ. Therefore, the DSS strength and hence the entropy jump across the DSS are different in the two cases; consequently states $B_{D}$ and $B_{D}^{\prime}$ are located on two different isentropes. Thus, the existence of a second DSS shock $\mathrm{A}_{\mathrm{D}}^{\prime}-\mathrm{B}_{\mathrm{D}}^{\prime}$ would lead to multiple solutions of the Riemann problem from state $\left(P_{1}, v_{1}\right)$ to $v_{2}$, since $P_{2}=P\left(s_{\mathrm{B}_{\mathrm{D}}}, v_{2}\right) \neq P\left(s_{\mathrm{B}_{\mathrm{D}}^{\prime}}, v_{2}\right)=P_{2^{\prime}}$.

The uniqueness of the double sonic rarefaction shock along a given isentrope is therefore guaranteed by the uniqueness of the solution of the Riemann problem.

In this respect, Liu (1975) demonstrated that there exists a unique solution to the Riemann problem of gasdynamics provided that

$$
\left(\frac{\partial P}{\partial e}\right)_{v}>0 \quad \text { and } \quad\left(\frac{\partial P}{\partial v}\right)_{e}<0
$$




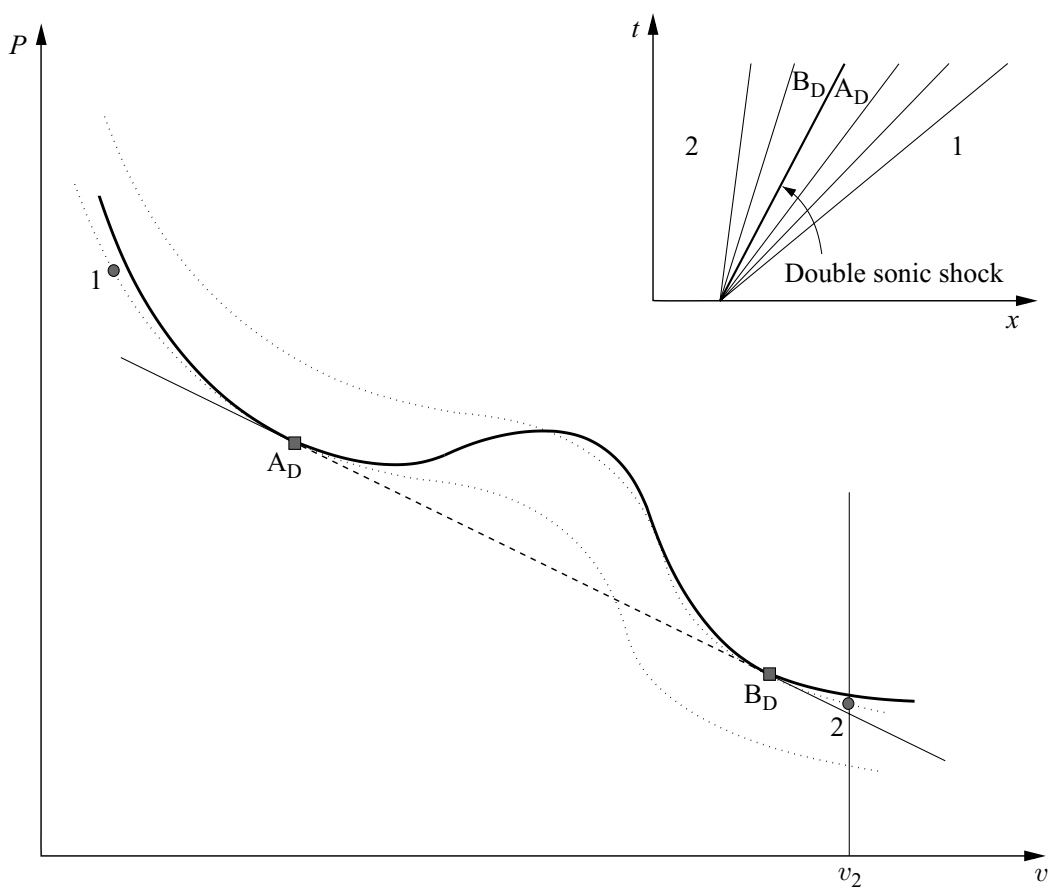

FIGURE 8. Rarefaction fan-shock-fan combination including a double sonic rarefaction shock.

The fulfilling of conditions (2.7) in the case of the dense gases considered here is detailed in the following. For all gases, the coefficient of thermal expansion is always positive, that is,

$$
\beta=\frac{1}{v}\left(\frac{\partial v}{\partial T}\right)_{P}>0
$$

Note that this condition is violated only in special cases, e.g. $\beta<0$ for water at freezing point. Therefore, using the cyclic derivative rule, one has

$$
\left(\frac{\partial P}{\partial T}\right)_{v}=-\left(\frac{\partial P}{\partial v}\right)_{T}\left(\frac{\partial v}{\partial T}\right)_{P}>0
$$

since the isothermal compressibility $k_{T}=-(1 / v)(\partial v / \partial P)_{T}$ is also a positive quantity for all substances. From the chain derivative rule, one finally has

$$
\left(\frac{\partial P}{\partial e}\right)_{v}=\left(\frac{\partial P}{\partial T}\right)_{v}\left(\frac{\partial T}{\partial e}\right)_{v}=\frac{1}{c_{v}}\left(\frac{\partial P}{\partial T}\right)_{v}>0,
$$

where $c_{v}=(\partial e / \partial T)_{v}>0$ is the isochoric specific heat. The first condition of (2.7) is therefore demonstrated. Considering now the second condition in (2.7), from the chain rule one has

$$
\left(\frac{\partial P}{\partial v}\right)_{e}=\left(\frac{\partial P}{\partial T}\right)_{v}\left(\frac{\partial T}{\partial v}\right)_{e}+\left(\frac{\partial P}{\partial v}\right)_{T} .
$$

By using the cyclic derivative rule and the definition of the isochoric specific heat, one obtains $(\partial T / \partial v)_{e}=-(\partial e / \partial v)_{T} / c_{v}$. By recalling the reciprocity relation, 
$(\partial e / \partial v)_{T}=T(\partial P / \partial T)_{v}-P$, see Emanuel (1987), one finally obtains

$$
\left(\frac{\partial P}{\partial v}\right)_{e}=\left(\frac{\partial P}{\partial v}\right)_{T}-\frac{T}{c_{v}}\left(\frac{\partial P}{\partial T}\right)_{v}\left[\left(\frac{\partial P}{\partial T}\right)_{v}-\frac{P}{T}\right] .
$$

In the above expression, $(\partial P / \partial v)_{T}<0$ since $k_{T}>0$ and $(\partial P / \partial T)_{v}>0$ from (2.9). Therefore, the condition

$$
\left(\frac{\partial P}{\partial T}\right)_{v} \geqslant \frac{P}{T}
$$

is a sufficient condition for (2.7) to hold. Note that for an ideal gas $(\partial P / \partial T)_{v} \equiv P / T$ and condition (2.10) is always satisfied. A noticeable example of a real gas model fulfilling the condition above is given by the van der Waals equations of state, $P(T, v)=$ $R T /(v-b)-a / v^{2}$, with $a, b$ and $R$ (positive) gas-dependent constants, for which

$$
\left(\frac{\partial P}{\partial T}\right)_{v}=\frac{R}{v-b}>\frac{P}{T}=\frac{R}{v-b}-\frac{a}{T v^{2}} .
$$

A more general result is obtained by recalling the definition of the compressibility factor,

$$
Z(T, v)=\frac{P(T, v) v}{R T}
$$

which can be used to recast the pressure equation of state as $P(T, v)=Z(T, v) R T / v$. Therefore, in terms of the compressibility factor $Z$, condition (2.10) reads

$$
\left(\frac{\partial Z}{\partial T}\right)_{v} \geqslant 0
$$

Condition (2.12) holds for the dense gas states, namely for $T_{r}=T / T_{\mathrm{c}} \lesssim 1.7$ and $P_{r}=P / P_{\mathrm{c}} \lesssim 5$. This feature can be appreciated from figure $9(b)$, where several isochores for argon are shown as a function of $Z$ and $T_{r}$ in the region of interest, that is for $v_{r} \geqslant 1$. Figure $9(a)$ reports the common compressibility $Z-P_{r}$ chart of argon in order to highlight the thermodynamic region of interest. As is well-known from experiments, the compressibility charts of any fluid are similar from both a qualitative and a quantitative point of view. This empirical law is known as the principle of correspondent states. Therefore the compressibility chart represents a useful tool with which to discuss general thermodynamic properties regardless of the particular substance under consideration, see e.g. Callen (1985).

To conclude, provided that conditions (2.8) and (2.10) or, equivalently, (2.12) are fulfilled, as is the case for the dense gases considered in this work, the Riemann problem has a unique solution and hence, on a given isentrope, only one state point leading to a DSS exists.

Thermodynamic states from which a DSS originates can be computed as follows. For a given value $\bar{s}$ of the entropy, the pre-shock and post-shock states connected by the DSS are computed by solving the system of four equations

$$
\left.\begin{array}{rl}
P_{\mathrm{A}_{\mathrm{D}}} & =P\left(\bar{s}, v_{\mathrm{A}_{\mathrm{D}}}\right), \\
c\left(P_{\mathrm{A}_{\mathrm{D}}}, v_{\mathrm{A}_{\mathrm{D}}}\right) / v_{\mathrm{A}_{\mathrm{D}}} & =c\left(P_{\mathrm{B}_{\mathrm{D}}}, v_{\mathrm{B}_{\mathrm{D}}}\right) / v_{\mathrm{B}_{\mathrm{D}}}, \\
P^{\mathrm{H}}\left(v_{\mathrm{B}_{\mathrm{D}}} ; P_{\mathrm{A}_{\mathrm{D}}}, v_{\mathrm{A}_{\mathrm{D}}}\right) & =P^{\mathrm{R}}\left(v_{\mathrm{B}_{\mathrm{D}}} ; P_{\mathrm{A}_{\mathrm{D}}}, v_{\mathrm{A}_{\mathrm{D}}}, c\left(P_{\mathrm{A}_{\mathrm{D}}}, v_{\mathrm{A}_{\mathrm{D}}}\right)\right), \\
P_{\mathrm{B}_{\mathrm{D}}} & =P^{\mathrm{R}}\left(v_{\mathrm{B}_{\mathrm{D}}} ; P_{\mathrm{A}_{\mathrm{D}}}, v_{\mathrm{A}_{\mathrm{D}}}, c\left(P_{\mathrm{A}_{\mathrm{D}}}, v_{\mathrm{A}_{\mathrm{D}}}\right)\right),
\end{array}\right\}
$$


(a)

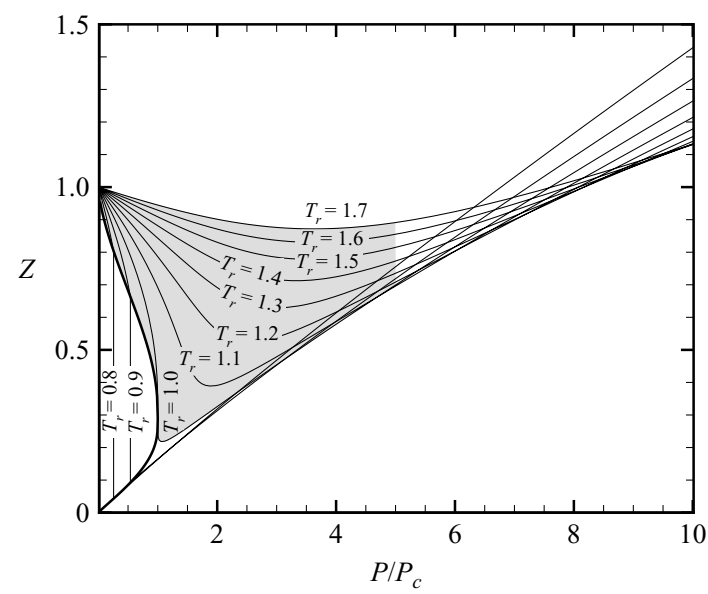

(b)

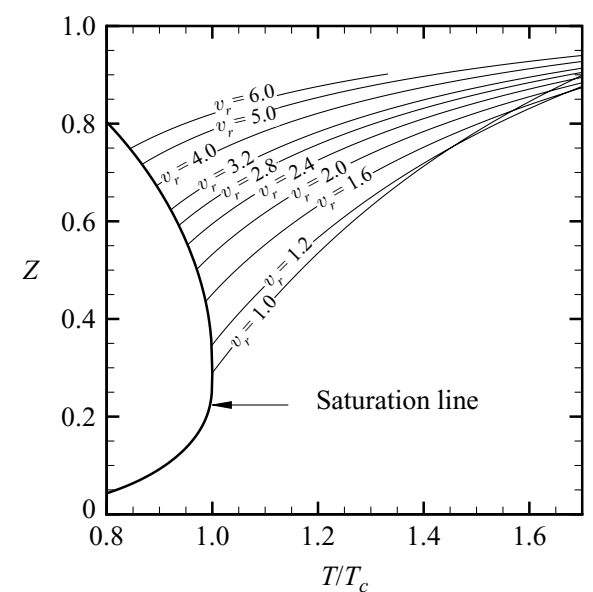

FIGURE 9. Compressibility charts for argon calculated with the thermodynamic model of Tegeler, Span \& Wagner (1999). (a) The dense gas region of interest is highlighted in the more usual $Z-P_{r}$ chart, $(b)$ isochors $(-)$ as a function of $Z$ and $T_{r}$ in the dense gas region have positive slope.

for the unknown specific volume and pressure at state points $A_{D}=A_{D}(\bar{s})$ and $\mathrm{B}_{\mathrm{D}}=\mathrm{B}_{\mathrm{D}}(\bar{s})$. In $(2.13) c(P, v)$ represents the sound speed as a function of pressure and specific volume. Note that the post-shock state point $\mathrm{B}_{\mathrm{D}}(\bar{s})$ lies on an isentrope with $s>\bar{s}$. The first equation of (2.13) guarantees that state $\mathrm{A}_{\mathrm{D}}$ lies on the $s=\bar{s}$ isentrope; the remaining three equations are equivalent to the Rankine-Hugoniot system (2.1) for sonic pre- and post- shock states, see $\S 2.1$. System (2.13) has no solution for all isentropes having $s>s_{\tau}$, namely if $\Gamma$ is always positive along the isentrope.

By letting the value of the entropy change, states $\mathrm{A}_{\mathrm{D}}(s)$ from which a DSS can originate, together with the corresponding post-shock states $\mathrm{B}_{\mathrm{D}}(s)$, describe a oneparameter curve which will be referred to as the double sonic locus (DSL) in the following. Cramer \& Sen (1987) derived an analytical expression for double sonic states in the case of a polytropic van der Waals gas, i.e. of the DSL, which is reported here for completeness:

$$
\pi^{\mathrm{D}}(\nu)=\frac{\left[(1-\delta)^{2}-(1-\delta)(1-3 \delta) / v-8 \delta / v^{2}-4 / \nu^{3}\right] / v}{2(1+\delta)(2+\delta)}
$$

where $\pi=P /\left(a / b^{2}\right)$ and $v=v / b$ are pre-shock values and where $\delta=R / c_{v}$, with $c_{v}$ being a constant according to the polytropic approximation. In the next section, the DSL is used as the boundary of the thermodynamic region in which RSWs are admissible and other aspects are discussed.

\section{The rarefaction shocks region of dense gases}

The results from the previous sections are used in the following to identify the thermodynamic region in which rarefaction shocks are admissible.

Pre-shock states on an isentrope which does not cross the liquid-vapour saturation curve are considered first, as in $\S 2.2$. The double sonic shock states $A_{D}$ and $B_{D}$ are computed together with the intersections of the isentrope and the $\Gamma=0$ line, which are denoted by $\Gamma_{0,1}$ and $\Gamma_{0,2}$, with $\Gamma_{0,1}$ being associated with the lowest specific volume. 


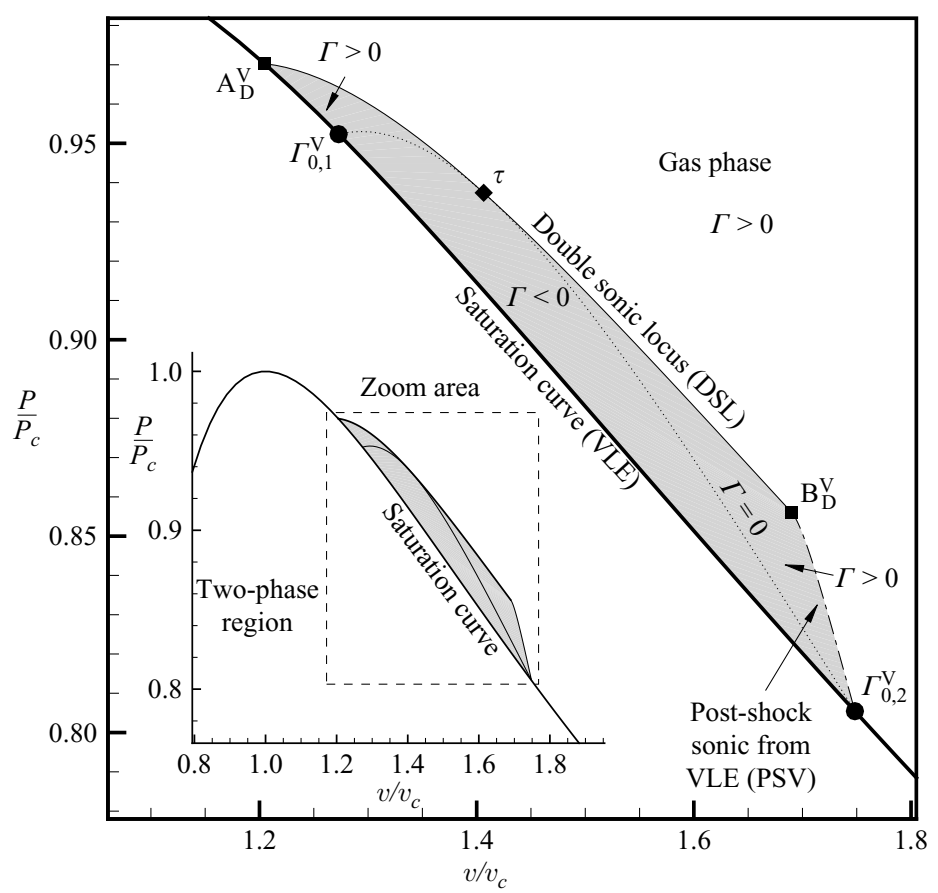

FIGURE 10. Rarefaction shocks region (RSR) for a van der Waals polytropic gas with $R / c_{v}=0.052$.

For pre-shock states with specific volume lower than that of state $A_{D}$, no RSW are possible. From state $A_{D}$, the only admissible RSW is the DSS connecting $A_{D}$ and $\mathrm{B}_{\mathrm{D}}$, as in figure 5. Between states $\mathrm{A}_{\mathrm{D}}$ and $\Gamma_{0,1}$, either pre-shock sonic or post-shock sonic RSW are possible, together with non-sonic RSW. Note that the specific volume of the post-shock states ranges from the value corresponding to the pre-shock sonic RSW up to the one corresponding to the post-shock sonic RSW, see figure 6. For pre-shock states embedded in the $\Gamma<0$ region, namely, between $\Gamma_{0,1}$ and $\Gamma_{0,2}$, only post-shock sonic and non-sonic RSW are possible. Post-shock specific volumes are limited by the pre-shock value - corresponding to an RSW of zero intensity, i.e. to an acoustic wave - up to the post-shock sonic state. For specific volumes larger than that associated with $\Gamma_{0,2}$, no RSW are possible regardless of the post-shock state.

Following the above considerations, a rarefaction shock region (RSR) can be defined as the one which embeds all the thermodynamic states that can possibly be connected by a RSW. The newly defined thermodynamic region is depicted in figure 10 , for a van der Waals polytropic gas with $R / c_{v}=0.052$. The DSL is split into a pre-shock portion identifying all states $A_{D}$, lying along the curve from $A_{D}^{v}$ to $\tau$, and a corresponding post-shock line from $\tau$ to $\mathrm{B}_{\mathrm{D}}^{\mathrm{V}}$. Point $\mathrm{A}_{\mathrm{D}}^{\mathrm{V}}$ is the intersection of the DSL and the VLE line; the latter is taken as an additional boundary of the RSR since only single-phase RSW are considered here. All shocks originating from the VLE line, from $\mathrm{A}_{\mathrm{D}}^{\mathrm{V}}$ to the point $\Gamma_{0,2}^{\mathrm{V}}$, where the $\Gamma=0$ curve intersects the VLE line, have a maximum specific volume at the post-shock state which corresponds to the post-shock sonic conditions. These limiting post-shock states constitute the last portion of the boundary of the RSR, namely, the post-shock sonic from VLE (PSV) line indicated in figure 10. 


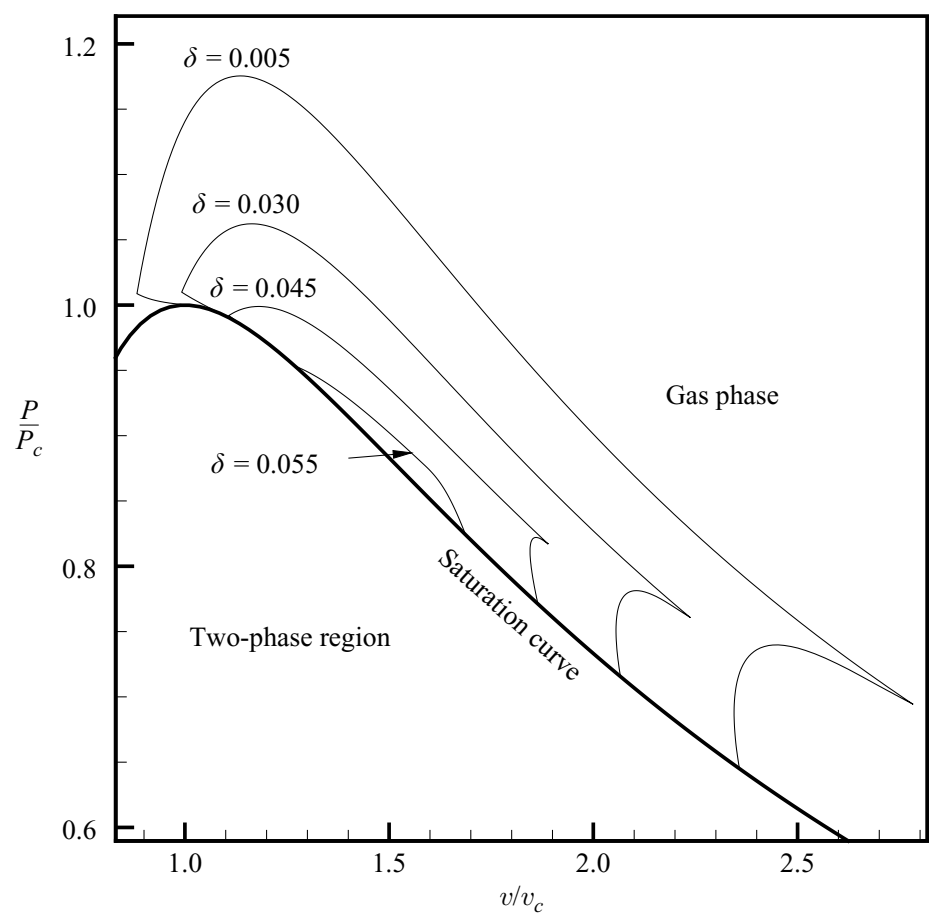

FIGURE 11. Rarefaction shock region (RSR) for different van der Waals polytropic gases; $\delta=R / c_{v}$.

Remarkably, the RSR is larger than the $\Gamma<0$ region, as expected from previous studies, and it also includes states with $\Gamma>0$. In the following section, the influence of molecular complexity on the shape and extent of the RSR is investigated. Finally, an accurate multi-parameter thermodynamic model is used to verify the qualitative results obtained here by means of the simple polytropic van der Waals model.

\subsection{Influence of molecular complexity}

Different fluids are considered to study the influence of molecular complexity on the shape and extent of the rarefaction shock region defined in the previous section.

Figure 11 shows the RSR of fluids with increasing molecular complexity for a van der Waals polytropic gas. The only parameter which characterizes the molecular complexity in such a gas is the dimensionless (inverse) specific heat $\delta=R / c_{v}$. In particular, the lower the value of $\delta$, the higher the molecular complexity of the fluid, see Colonna \& Guardone (2006). Accordingly, different curves in figure 11 correspond to different values of $\delta$.

The size of the RSR increases with increasing molecular complexity, as does the size of the $\Gamma<0$ region. The shape of the RSR is that depicted in figure 10 up to the special value $\delta \simeq 0.05105$. For $\delta>0.05105$, the Rankine-Hugoniot curve connecting the limiting states $\mathrm{A}_{\mathrm{D}}^{\mathrm{V}}$ and $\mathrm{B}_{\mathrm{D}}^{\mathrm{V}}$ crosses the liquid-vapour equilibrium region. Initial states which lead to the crossing of the VLE line can be removed from the RSR region by computing a limiting Rankine-Hugoniot curve originating from the DSL line that is tangent to the saturation curve, as shown in figure 12. The additional boundary of the RSR region is the locus of all pre-shock states whose associated RankineHugoniot curve are tangent to the saturation curve. Note that such an enlarged RSR represents an extreme situation not likely to be observed in real substances. In fact, 


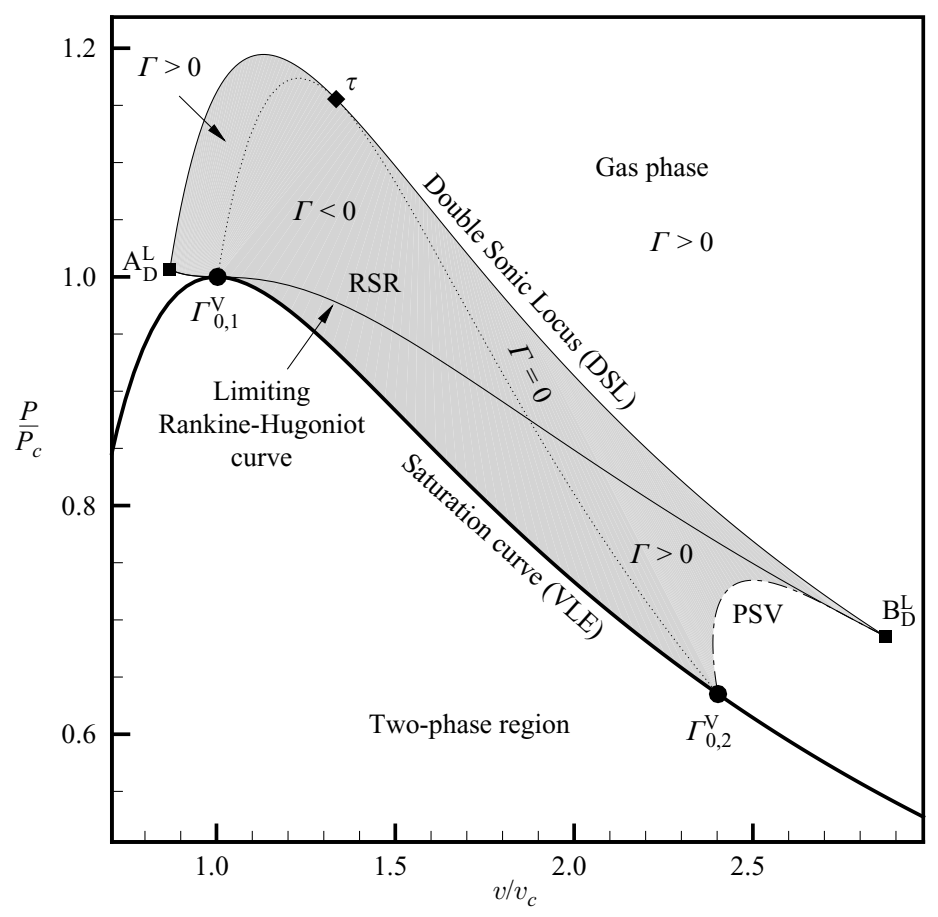

FIGURE 12. Rarefaction shock region (RSR) for a van der Waals polytropic gas with $R / c_{v}=0.001$.

it is well known that the van der Waals model predicts a $\Gamma<0$ region, and hence an RSR, which is larger if compared to more accurate thermodynamic models, as discussed for example by Cramer (1989) and Guardone \& Argrow (2005). In the next section, the qualitative information obtained here by means of the simple van der Waals model is confirmed by the more accurate predictions obtained by means of multi-parameter thermodynamic models.

\subsection{Span-Wagner model of siloxane fluids}

The simple van der Waals model used in the previous sections to determine the RSR is abandoned here in favour of more complex multi-parameter equations of state in the Span-Wagner form, see e.g. Span \& Wagner $(2003 a, b)$. This modern equation of state allows accurate computations of all relevant thermodynamic properties over the entire thermodynamic range (even close to the critical point) with the accuracy required for design and analysis of advanced technical applications. Recently, 12-parameter equations of state of the Span-Wagner type for selected linear and cyclic siloxanes have become available, see Colonna et al. (2006) and Colonna, Nannan \& Guardone (2008b), and siloxanes were proposed as candidate BZT fluids, see Colonna \& Silva (2003) and Colonna, Guardone \& Nannan (2007). These fluids are light silicon oils currently used in Organic Rankine Cycle turbomachinery applications and considered as potential candidates for the experimental verification of the existence of nonclassical phenomena, see Zamfirescu et al. (2006a, b) and Colonna et al. (2008a).

Figure 13 shows the RSR of the cyclic siloxane $\mathrm{D}_{6}\left(\mathrm{C}_{12} \mathrm{H}_{36} \mathrm{O}_{6} \mathrm{Si}_{6}\right)$ and of the linear siloxane $\mathrm{MD}_{4} \mathrm{M}\left(\mathrm{C}_{14} \mathrm{H}_{42} \mathrm{O}_{5} \mathrm{Si}_{6}\right)$, computed by means of the mentioned multi-parameter equations of state. The qualitative trends obtained in the previous section with the van der Waals model are therefore confirmed by the more complex Span-Wagner 

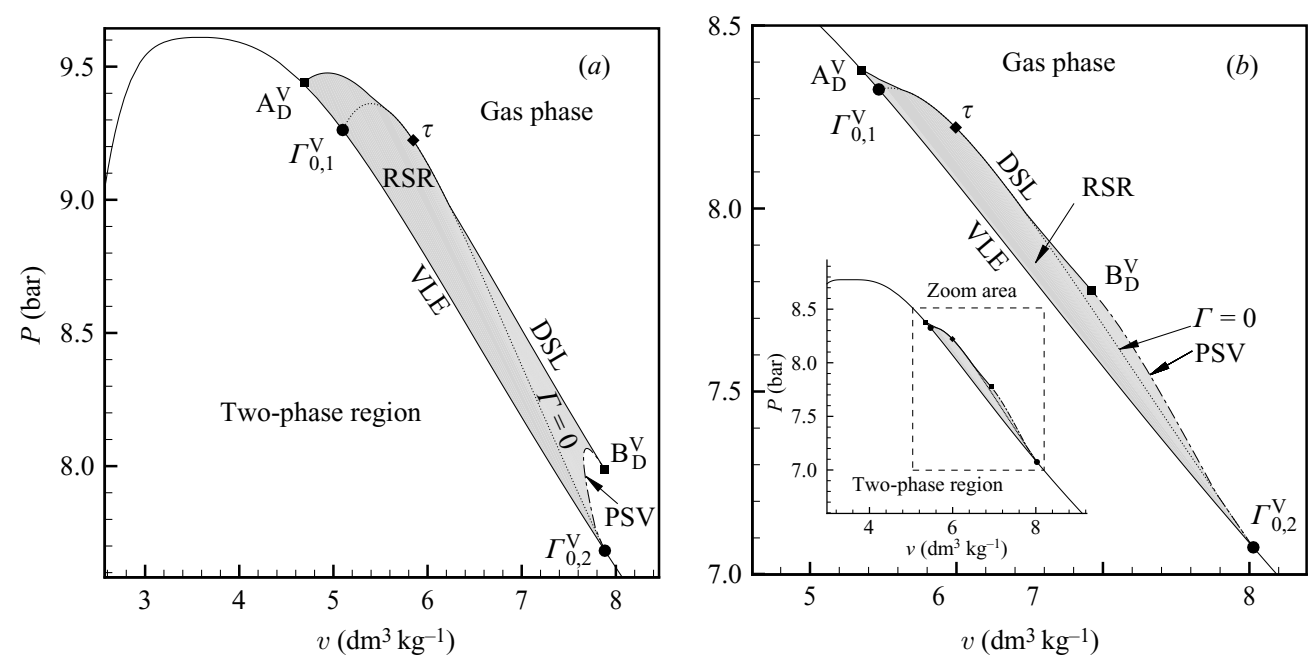

FIGURE 13. RSR computed using the multi-parameter equations of state: $(a)$ cyclic siloxane fluid $\mathrm{D}_{6}$ (Dodecamethyl-cyclohexasiloxane, $\mathrm{C}_{12} \mathrm{H}_{36} \mathrm{O}_{6} \mathrm{Si}_{6}$ ), (b) linear siloxane fluid $\mathrm{MD}_{4} \mathrm{M}$ (Tetradecamethyl-hexasiloxane, $\mathrm{C}_{14} \mathrm{H}_{42} \mathrm{O}_{5} \mathrm{Si}_{6}$ ).

model. Note that, even for the most complex siloxane fluids, the conditions depicted in figure 12 are never encountered and all plots qualitatively resemble the results shown in figure 10. To conclude, as first noticed by Thompson \& Lambrakis (1973), it should be recalled that the value of $\Gamma$ and therefore the shape and size of the RSR for a given fluid strongly depends on the thermodynamic model used to describe the fluid properties, whose accuracy is to be validated against experimental results, see Colonna et al. (2007).

\section{Conclusions}

A new thermodynamic region is introduced to circumvent the limits of the currently accepted definition of the non-classical region, which relies exclusively upon the sign of the fundamental derivative of gasdynamics $\Gamma$.

The rarefaction shocks region, which also encompasses thermodynamic states with $\Gamma>0$, is bounded by the double sonic locus and the liquid-vapour equilibrium curve and embeds the $\Gamma<0$ region. The rarefaction shocks region embeds all the thermodynamic states which can possibly be connected by a rarefaction shock.

The existence of only one double sonic shock along a given isentrope is demonstrated for dense gases and therefore the double sonic locus is a curve in the volume-pressure thermodynamic plane.

Results include the computation of the rarefaction shocks region obtained with the simple polytropic gas model, but the numerical procedure is also applied using the multi-parameter Span-Wagner thermodynamic model for siloxanes. Although the extent of the rarefaction shocks region is found to be strongly dependent on the thermodynamic model of the fluid, the qualitative trend given by the van der Waals model is confirmed by the more accurate Span-Wagner equation of state.

The correct determination of the size and shape of the thermodynamic region in which non-classical phenomena can possibly occur, namely the definition of the rarefaction shocks region introduced here, is key to the design of applications 
exploiting non-classical gasdynamic effects and to the experimental investigations of non-classical phenomena in the dense gas region.

This research is supported by the Dutch Technology Foundation STW, Applied Science Division of NWO and the Technology Program of the Ministry of Economic Affairs, DSF 6573. The authors acknowledge the contribution of their colleague and friend Ryan Nannan for discussions on thermodynamic models.

\section{REFERENCES}

Bethe, H. A. 1942 The theory of shock waves for an arbitrary equation of state. Tech. Rep. 545. Office of Scientific Research and Development.

Callen, H. B. 1985 Thermodynamics and an Introduction to Thermostatistics, 2nd edn. Wiley.

Colonna, P. \& Guardone, A. 2006 Molecular interpretation of non-classical gasdynamics of dense vapors under the van der waals model. Phys. Fluids 18, 056101-1-14.

Colonna, P., Guardone, A. \& Nannan, N. R. 2007 Siloxanes: a new class of candidate BetheZel'dovich-Thompson fluids. Phys. Fluids 19, 086102-1-12.

Colonna, P., Guardone, A., Nannan, N. R. \& Zamfirescu, C. 2008 a Design of the dense gas flexible asymmetric shock tube. Trans. ASME: J. Fluids Engng (In press).

Colonna, P., NANnAN, N. R. \& Guardone, A. $2008 b$ Multiparameter equations of state for siloxanes: $\left[\left(\mathrm{CH}_{3}\right)_{3}-\mathrm{Si}-\mathrm{O}_{1 / 2}\right]_{2}-\left[\mathrm{O}-\mathrm{Si}-\left(\mathrm{CH}_{3}\right)_{2}\right]_{\mathrm{i}=1 \ldots 3}$, and $\left[\mathrm{O}-\mathrm{Si}-\left(\mathrm{CH}_{3}\right)_{2}\right]_{6}$. Fluid Phase Equilib. 263, 115-130.

Colonna, P., Nannan, R., Guardone, A. \& Lemmon, E. W. 2006 Multiparameter equations of state for selected siloxanes. Fluid Phase Equilib. 244, 193-211.

Colonna, P. \& Silva, P. 2003 Dense gas thermodynamic properties of single and multicomponent fluids for fluid dynamics simulations. Trans. ASME: J. Fluids Engng 125, 414-427.

Cramer, M. S. 1989 Negative nonlinearity in selected fluorocarbons. Phys. Fluids A 1, 1894-1897.

Cramer, M. S. \& Sen, R. 1987 Exact solutions for sonic shocks in van der Waal's gases. Phys. Fluids 30, 377-385.

Emanuel, G. 1987 Advanced Classical Thermodynamics. AIAA Education Series.

Fergason, S. H., Ho, T. L., Argrow, B. M. \& Emanuel, G. 2001 Theory for producing a single-phase rarefaction shock wave in a shock tube. J. Fluid Mech. 445, 37-54.

Godlewski, E. \& Raviart, P. A. 1994 Numerical Approximation of Hyperbolic Systems of Conservation Laws. Springer.

Guardone, A. \& ARgrow, B. M. 2005 Non-classical gasdynamic region of selected fluorocarbons. Phys. Fluids 17, 116102-1-17.

HAYES, W. 1960 The basic theory of gasdynamic discontinuities. In Fundamentals of Gasdynamics (ed. H. W. Emmons). High speed aerodynamics and jet propulsion, vol. 3, pp. 416-481. Princeton University Press.

KLuwick, A. 2001 Theory of shock waves. Rarefaction shocks. In Handbook of Shockwaves (ed. G. Ben-Dor, O. Igra, T. Elperin \& A. Lifshitz), vol. 1, chap. 3.4, pp. 339-411. Academic.

Landau, L. D. \& Lifshitz, E. M. 1959 Course of Theoretical Physics, Fluid Mechanics, vol. 6. Pergamon.

Lax, P. 1957 Hyperbolic systems of conservation laws, II. Commun. Pure Appl. Maths 10, 537-566.

LiU, T. P. 1975 The Riemann problem for general systems of conservation laws. J. Diff Equat. 18, 218-234.

OLEINIK, O. 1959 Uniqueness and stability of the generalized solution of the Cauchy problem for a quasilinear equation. Uspehi Mat. Nauk 14, 165-170.

Smoller, J. 1983 Shock Waves and Reaction-Diffusion Equations. Springer.

Span, R. \& WaGner, W. $2003 a$ Equations of state for technical applications. I. simultaneously optimized functional forms for nonpolar and polar fluids. Intl J. Thermophys. 24, 1-39.

SPAN, R. \& WaGNER, W. $2003 b$ Equations of state for technical applications. II. Results for nonpolar fluids. Intl J. Thermophys. 24, 41-109.

Tegeler, C., Span, R. \& Wagner, W. 1999 A new equation of state for Argon covering the fluid region for temperatures from the melting line to $700 \mathrm{~K}$ at pressures up to $1000 \mathrm{MP}$. J. Phys. Chem. Ref. Data 28, 779-850. 
Thompson, P. A. 1971 A fundamental derivative in gas dynamics. Phys. Fluids 14, 1843-1849.

Thompson, P. A. \& Lambrakis, K. C. 1973 Negative shock waves. J. Fluid Mech. 60, 187-208.

Zamfirescu, C., Guardone, A. \& Colonna, P. $2006 a$ Numerical simulation of the FAST dense gas Ludwieg tube experiment. In ECCOMAS CFD 2006 Conference, Egmond aan Zee, $N L$.

Zamfirescu, C., Guardone, A. \& Colonna, P. $2006 b$ Preliminary design of the FAST dense gas Ludwieg tube. In 9th AIAA/ASME Joint Thermophysics and Heat Transfer Conference, San Francisco, $C A$.

Zel'dovich, Y. B. 1946 On the possibility of rarefaction shock waves. Zh. Eksp. Teor. Fiz. 4, 363-364. 